\title{
Submucosal esophageal hematomas in a critically ill patient on anticoagulation
}

\author{
Hassam Ali $^{{ }^{*}}{ }^{(0)}$, Maliha Naseer ${ }^{2}$ (1) \\ ${ }^{1}$ Department of Internal Medicine, East Carolina University/Vidant Medical Center, Greenville, North Carolina 27834, USA \\ ${ }^{2}$ Department of Gastroenterology, East Carolina University/Vidant Medical Center, Greenville, North Carolina 27834, USA
}

*Correspondence: Hassam Ali, Department of Internal Medicine, East Carolina University/Vidant Medical Center, Greenville, North Carolina 27834, USA. Alih20@ecu.edu

Academic Editor: Amedeo Lonardo, Azienda Ospedaliero Universitaria di Modena, Italy

Received: May 29, 2021 Accepted: July 22, 2021 Published: August 31, 2021

Cite this article: Ali H, Naseer M. Submucosal esophageal hematomas in a critically ill patient on anticoagulation. Explor Med. 2021;2:343-7. https://doi.org/10.37349/emed.2021.00052

\begin{abstract}
Submucosal esophageal hematoma is an uncommon clinical complication of anticoagulation. Current literature is scarce regarding presentation and management in acute submucosal hematomas in critically ill patients. Patients often present with retrosternal chest pain, making the diagnosis challenging due to overlap with common presentations of cardiopulmonary disorders. A high degree of suspicion is necessary in sedated patients. Several factors contribute to its etiology, and diagnosis often requires invasive techniques like endoscopy. However, management is usually supportive and aimed at its underlying cause. This is a case of a 68-year-old female who developed submucosal esophageal hematomas following anticoagulation for subarachnoid hemorrhage-related delayed neurological deficits in the intensive care unit.
\end{abstract}

\section{Keywords}

Submucosal esophageal hematoma, anticoagulation, subarachnoid hemorrhage, delayed neurological deficits

\section{Introduction}

The pathophysiology of rarely reported submucosal esophageal hematomas involves injury resulting in rupture of blood vessels in the submucosal layer resulting in hemorrhage and hematoma formation [1]. Submucosal esophageal hematomas should be differentiated from other etiologies which present as chest pain, as that remains the most common presentation. Several predisposing factors contribute to their formation, including trauma secondary to medical apparatus, coagulation disorders, and anticoagulation $[2,3]$. This is a case of submucosal esophageal hematomas after initiation of anticoagulant therapy for subarachnoid hemorrhage related delayed neurological deficits.

\section{Case report}

A 68-year-old female presented to the emergency department with the progressive left frontal headache of one week, nausea, vomiting, photophobia and diplopia. Her previous medical history was significant for alcohol

(C) The Author(s) 2021. This is an Open Access article licensed under a Creative Commons Attribution 4.0 International License (https://creativecommons.org/licenses/by/4.0/), which permits unrestricted use, sharing, adaptation, distribution and reproduction in any medium or format, for any purpose, even commercially, as long as you give appropriate credit to the original author(s) and the source, provide a link to the Creative Commons license, and indicate if changes were made. 
abuse, hepatitis $\mathrm{C}$ infection, and tobacco abuse. The patient had undergone head computed tomography (CT) scan the same morning, which was concerning for a subarachnoid hemorrhage (SAH). On presentation, her blood pressure was $125 / 68 \mathrm{mmHg}$, the pulse of 74 beats/min, and a temperature of $97.5^{\circ} \mathrm{F}$. On physical examination, significant findings were got included a Glasgow Coma Scale of 14 when the patient was able to move all extremities, vocalized and eyes opened to command. The pupils were asymmetrical with 3rd cranial nerve palsy in the left eye as the patient had a loss of outward movement. Her gross motor strength was reduced to $4 / 5$ on the right side with intact gross motor strength on the left. Due to continued episodes of vomiting, she was intubated for airway protection. An emergent head CT was concerning for an aneurysm in the left prepontine cistern and computed tomography angiography (CTA) displayed subarachnoid hemorrhage throughout basal cisterns, and an $11 \mathrm{~mm}$ left posterior communicating artery aneurysm.

An external ventricular drain was placed to relieve intracranial pressure, and the patient underwent successful balloon-assisted coil embolization of the aneurysm. A heparin drip at $8 \mathrm{IU} / \mathrm{kg}$ per hour for SAH-induced delayed neurological deficits was started. Two days post antithrombotic therapy; the patient had an occurrence of $200 \mathrm{~mL}$ bright red blood coming out of the orogastric tube. Her hemoglobin (Hb) on initial presentation was $12.7 \mathrm{~g} / \mathrm{dL}$ and trended down following two days after the start of anticoagulant therapy (Figure 1).

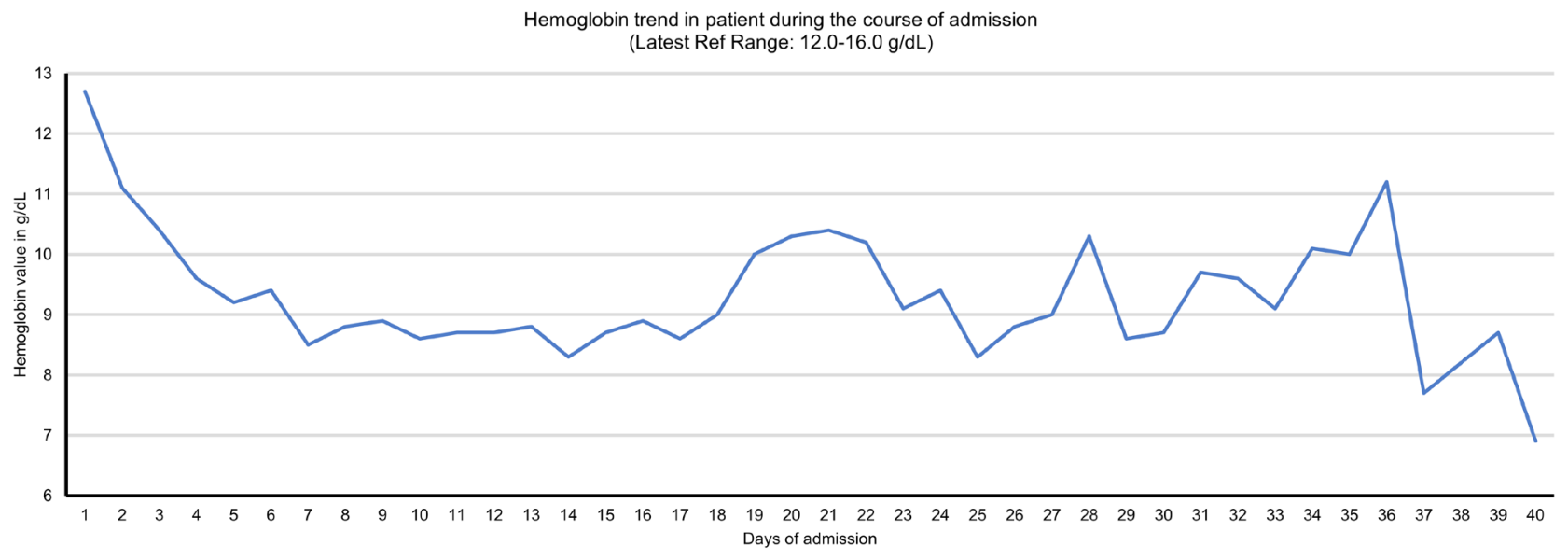

Figure 1. Hemoglobin trend in patient following anticoagulation therapy

Her activated partial thromboplastin time (aPTT) was elevated to more than 100 seconds. This was followed by hemodynamic instability requiring pressor support. Gastroenterology was consulted, and anticoagulation was held. The patient was started on proton pump inhibitors $40 \mathrm{mg}$ twice daily and received 1 unit of packed red blood cells and three units of fresh frozen plasma to reverse anticoagulation. Emergent endoscopy revealed Los Angeles Grade D esophagitis with a prominent segmental necrotic appearing mucosal protrusion in upper to mid-third of the esophagus (non-bleeding submucosal hematomas; Figure 2, Figure 3).

There was evidence of gastropathy in the proximal stomach, which was negative for intestinal metaplasia, dysplasia or malignancy except for a small benign hyperplastic polyp. The duodenum was normal in the examination. The decision was made to obtain an abdominal ultrasound with doppler to evaluate any evidence of cirrhosis and chest $\mathrm{x}$-ray to rule out esophageal perforation due to the history of continued vomiting. Chest x-ray was negative for any perforation, and an abdominal ultrasound revealed hepatic steatosis without cirrhosis. Further imaging was not considered due to limited influence on management and hemoglobin levels remained stable after transfusion as shown previously in Figure 1.

There were no further episodes of gastrointestinal bleed, and the patient was managed conservatively. Her hospital course was complicated by the continued decline in the neurological state with increased cardiopulmonary support resulting in uremia and metabolic acidosis. The patient was later started on continuous renal replacement therapy, but despite aggressive solute clearance, acidosis persisted, and 
hemodynamic status continued to decline. Goals of care discussion held with family resulted in a decision to pursue comfort care, and the patient was later pronounced dead.

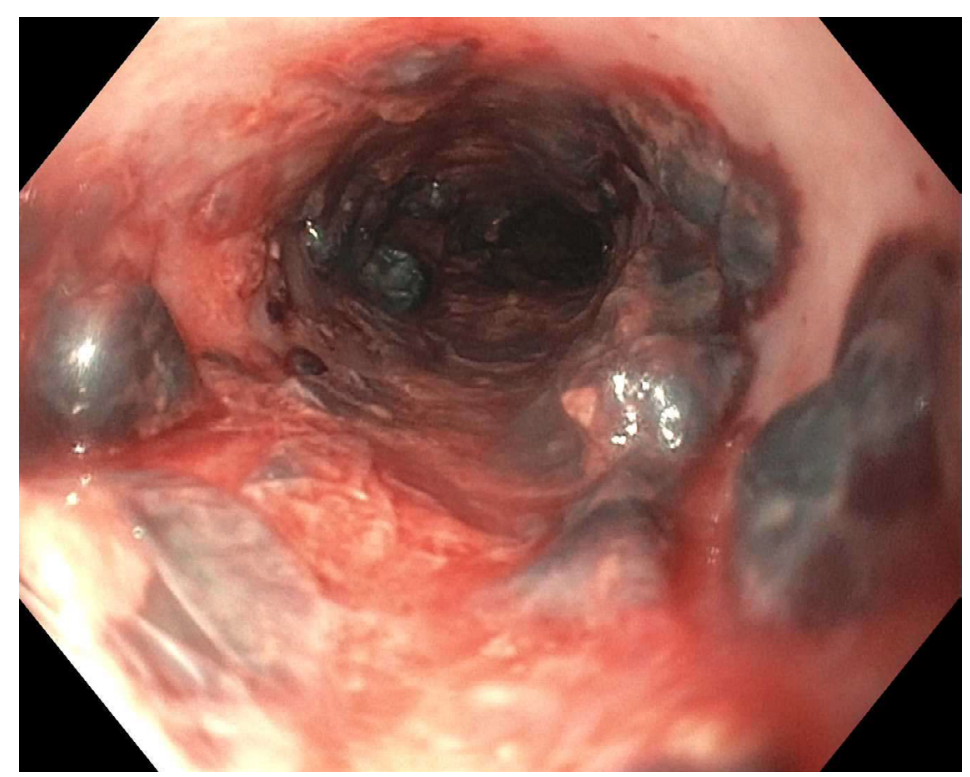

Figure 2. Necrotic mucosal protrusion in mid-third of the esophagus representing submucosal hematomas with orogastric tube extracted during the endoscopy

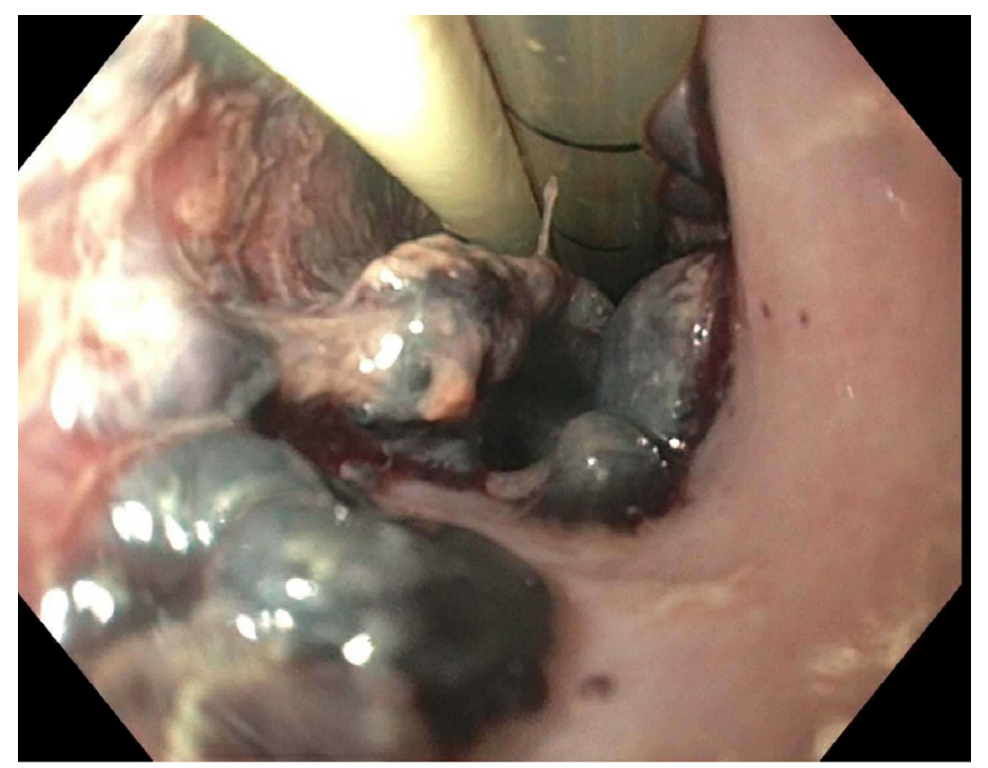

Figure 3. Necrotic mucosal protrusion in upper-third of the esophagus (endotracheal and orogastric tubes are also visualized)

\section{Discussion}

Submucosal esophageal hematomas have been categorized into spontaneous or traumatic [1]. The spontaneous etiology includes raised intra-esophageal pressure due to vomiting or retching, resulting in hemorrhage and hematomas in the blood vessels of the submucosal layer. Blood dyscrasias have also been reported to be the spontaneous cause behind submucosal hematomas. These include coagulopathy disorder such as hemophilia, hemodialysis, liver cirrhosis, antiplatelet or anticoagulant therapy [4]. Bleeding tendencies are often overlooked when considering etiologies for submucosal hematomas, and cases have reported anticoagulant use as a contributing factor, vessel fragility could be another possibility. However, change in prognosis and therapeutical approach are doubtful.

Traumatic factors include endoscopy, gastric tubes, bougies or any other medical apparatus [1]. Literature has hypothesized that submucosal trauma from the insertion of a medical apparatus could create a nidus for 
submucosal bleed, which is exaggerated when a patient is started on anticoagulant or antiplatelet therapy [5]. This could be another etiology of submucosal hematoma development in our patients as she did not have any history of previous bleeding or gastric varices. However, there was no reported resistant felt when the gastric tube was inserted.

Another classification for gastroesophageal submucosal hematomas would be, impaired hemostasis, emetogenic, food-induced, and spontaneous. Based on this proposed classification, our case would be explained by the impaired hematemesis than spontaneous as that is reserved for scenarios not justified by any other etiologies [6]. Initial presentation of a submucosal hematoma includes chest pain, vomiting, sometimes indistinguishable from acute chest syndrome. These are succeeded by hematemesis, although most reported blood loss is around $200 \mathrm{~mL}$, as in our case [7]. A submucosal hematoma is mostly diagnosed by endoscopy; a CT scan could be used to make a tentative diagnosis, especially if the hematoma is large and displacing surrounding structures, where it can show as an isodense area [1]. For cerebral aneurysms, coil embolization is followed by an anticoagulant to prevent any delayed neurological deficits [8]. However, in this study, endpoints like death were excluded. In addition, there are possible other exclusions and allocation biases. The guidelines are institutional dependent. Our patient was also started on 8 unit/kg per hour drip of unfractionated heparin. The intensive anticoagulant therapy should be considered as the etiology behind the development of submucosal hematomas, as in our case. However, this could be one of the contributing factors of her shock, although unlikely, considering the amount from the orogastric tube. Literature has pointed towards submucosal hemorrhage resulting in hemorrhagic shock requiring blood transfusion, the average hematemesis being $500 \mathrm{~g}$ or more [9]. A possible correlation between initiation of heparin therapy and development of esophageal hematomas exists, as described in our report. In addition, patients with the risk factors mentioned above (coagulopathies, iatrogenic trauma, etc.) may benefit from antiemetic therapy to prevent further esophageal injury in case of vomiting. However, more evidence is needed. Furthermore, our patient was intubated and sedated. Therefore, the role of antiemetic therapy is unsubstantiated. Limitations of our study include lacking confirmation of esophageal hematomas with CT scan chest.

We conclude that when starting antiplatelet or anticoagulant therapy after SAH, a cautious approach for risk factor assessment should be employed before starting anticoagulation therapy. The clinicians often use institutional or experience-based practice while starting anticoagulant therapy in the cerebrovascular intervention, especially due to the lack of evidence-based guidelines for antithrombotic therapy for delayed neurological deficits.

\section{Abbreviations}

CT: computed tomography

SAH: subarachnoid hemorrhage

\section{Declarations}

\section{Author contributions}

HA, MN contributed conception and design of the study; HA wrote the first draft of the manuscript. Both authors contributed to manuscript revision, read and approved the submitted version.

\section{Conflicts of interest}

The authors declare that they have no conflicts of interest.

\section{Ethical approval}

Not applicable.

\section{Consent to participate}

Informed consent to participate in the study was obtained from all participants or their surrogates. 


\section{Consent to publication}

Informed consent to publication was obtained from relevant participants.

Availability of data and materials

Not applicable.

\section{Funding}

Not applicable.

\section{Copyright}

(C) The Author(s) 2021.

\section{References}

1. Freeman AH, Dickinson RJ. Spontaneous intramural oesophageal haematoma. Clin Radiol. 1988;39:628-34.

2. Sanaka M, Kuyama Y, Hirama S, Nagayama R, Tanaka H, Yamanaka M. Spontaneous intramural hematoma localized in the proximal esophagus: truly “spontaneous"? J Clin Gastroenterol. 1998;27:265-6.

3. Hiller N, Zagal I, Hadas-Halpern I. Spontaneous intramural hematoma of the esophagus. Am J Gastroenterol. 1999;94:2282-4.

4. Sugimura K, Ishii N. Esophageal hematoma mimicking a large esophageal polyp: a diagnostic clue of acquired hemophilia A. Mayo Clin Proc. 2019;94:2142-3.

5. Ito S, Iwata S, Kondo I, Iwade M, Ozaki M, Ishikawa T, et al. Esophageal submucosal hematoma developed after endovascular surgery for unruptured cerebral aneurysm under general anesthesia: a case report. JA Clin Rep. 2017;3:54.

6. Sharma B, Lowe D, Antoine M, Shah M, Szyjkowski R. Intramural esophageal hematoma secondary to food ingestion. Cureus. 2019;11:e5623.

7. Keihanian S, Stoicov C, Bhattacharya K. Spontaneous intramural esophageal hematoma: a rare cause of chest pain, dysphagia and hematemesis. Am J Gastroenterol. 2011;106:S185.

8. Simard JM, Aldrich EF, Schreibman D, James RF, Polifka A, Beaty N. Low-dose intravenous heparin infusion in patients with aneurysmal subarachnoid hemorrhage: a preliminary assessment. J Neurosurg. 2013;119:1611-9.

9. Shim J, Jang JY, Hwangbo Y, Dong SH, Oh JH, Kim HJ, et al. Recurrent massive bleeding due to dissecting intramural hematoma of the esophagus: treatment with therapeutic angiography. World J Gastroenterol. 2009;15:5232-5. 